Real Analysis Exchange

Vol. 24(2), 1998/9, pp. 589-598

Jan Jastrzȩbski, Department of Mathematics, Gdańsk University, Wita Stwosza 57, 80-952 Gdańsk, Poland. e:mail jjas@ksinet.univ.gda.pl

Tomasz Natkaniec, Department of Mathematics, Gdańsk University, Wita Stwosza 57, 80-952 Gdańsk, Poland. e:mail: mattn@ksinet.univ.gda.pl

\title{
ON SUMS AND PRODUCTS OF EXTENDABLE FUNCTIONS
}

\begin{abstract}
We study the maximal additive, multiplicative and lattice-like families for the class of all extendable functions. This article is a continuation of earlier papers, in which the same questions concerning other Darboux-like functions have been studied.
\end{abstract}

\section{Preliminaries}

Our terminology is standard. By $\mathbb{R}$ and $\mathbb{I}$ we denote the set of all reals and the interval $[0,1]$, respectively. The letters $X, Y$ and $Z$ will denote topological spaces. The symbols $\bar{A}, \operatorname{int}(A)$ and $\operatorname{bd}(A)$ denote the closure, interior and boundary of a set $A$, respectively.

No distinction is made between a function and its graph. For functions $f: X \rightarrow Y$ and $g: X \rightarrow Z$, the symbol $(f, g)$ denotes the diagonal of $f$ and $g$, i.e., $(f, g): X \rightarrow Y \times Z,(f, g)(x)=(f(x), g(x))$ for every $x \in X$.

For a function $f: \mathbb{R} \rightarrow \mathbb{R}$ and $x \in \mathbb{R}$ the symbols $C^{-}(f, x), C^{+}(f, x)$ denote the cluster sets from the left and right, respectively, of the function $f$ at the point $x$.

By $\mathrm{C}(X, Y)$ and Const $(X, Y)$ we denote the family of all continuous functions and constant functions from $X$ to $Y$, respectively. We shall write $\mathrm{C}$ and Const when $X$ and $Y$ are clear from the context.

We shall consider the following classes of functions from $X$ to $Y$.

Key Words: extendable functions, peripherally continuous functions, family of peripheral intervals, maximal additive family, maximal multiplicative family.

Mathematical Reviews subject classification: Primary 26A15; Secondary 54C08.

Received by the editors April 16, 1998

*Both authors are supported by UG Research Grant No BW/5100-5-0282-7 and by NSF Cooperative Research Grant INT-9600548 with its Polish part financed by KBN. 
$\mathrm{PC}(X, Y)$ - the class of all peripherally continuous functions. A function $f: X \rightarrow Y$ is peripherally continuous if for every $x \in X$ and for all pairs of open sets $U$ and $V$ containing $x$ and $f(x)$, respectively, there exists an open subset $W \subset U$ such that $x \in W$ and $f(\operatorname{bd}(W)) \subset V$.

$\mathrm{E}(X, Y)-$ the class of all functions with property $\mathcal{E}$. A function $f: X \rightarrow Y$ has property $\mathcal{E}$ if it is extendable to a peripherally continuous function, i.e., if there exists a peripherally continuous function $F: X \times \mathbb{I} \rightarrow Y$ such that $f(x)=F(x, 0)$ for all $x \in X$.

$\operatorname{Conn}(X, Y)$ - the class of all connectivity functions. A function $f: X \rightarrow Y$ is connectivity, if the restriction $f\lceil C$ is a connected subset of $X \times Y$ whenever $C$ is a connected subset of $X$.

$\operatorname{Ext}(X, Y)$ - the family of extendable functions, i.e., functions $f: X \rightarrow Y$ for which there exists a connectivity function $F: X \times \mathbb{I} \rightarrow Y$ with property that $F(x, 0)=f(x)$ for every $x \in X$.

It is well-known that $\operatorname{PC}\left(\mathbb{R}^{2}, \mathbb{R}\right)=\operatorname{Conn}\left(\mathbb{R}^{2}, \mathbb{R}\right)$. (The inclusion " $C$ " was proved by Hamilton $[\mathrm{OH}]$ and by Stallings $[\mathrm{JS}]$, and the inclusion " $\supset$ " by Hagan $[\mathrm{H}]$.) Therefore, $\mathrm{E}(\mathbb{R}, \mathbb{R})=\operatorname{Ext}(\mathbb{R}, \mathbb{R})$.

Moreover, we shall consider the following class of real functions of one real variable that was introduced by $\mathrm{R}$. Fleissner $[\mathrm{RF}]$.

$\mathrm{M}$ - the class of all functions $f: \mathbb{R} \rightarrow \mathbb{R}$ such that if $x_{0}$ is a right (left) point of discontinuity of $f$, then $f\left(x_{0}\right)=0$ and there is a sequence $\left(x_{n}\right)$ converging to $x_{0}$ such that $x_{n}>x_{0}\left(x_{n}<x_{0}\right)$ and $f\left(x_{n}\right)=0$.

Recall that if $f \in \mathrm{M}$, then the set $D$ of all points at which $f$ is not continuous is nowhere dense, $D \subset f^{-1}(0)$, the set $f^{-1}(0)$ is closed, and $f$ is continuous on the closure of every component of $\mathbb{R} \backslash \operatorname{bd}\left(f^{-1}(0)\right)$. Consequently, $f$ is a Darboux Baire one function. Thus it is also an extendable function. (See $[\mathrm{BHL}]$.

Let $\mathcal{X}$ be a class of real functions. The maximal additive (multiplicative, lattice-like, respectively) class for $\mathcal{X}$ is defined to be the class of all $f \in \mathcal{X}$ for which $f+g \in \mathcal{X}(f g \in \mathcal{X}, \max (f, g) \in \mathcal{X}$ and $\min (f, g) \in \mathcal{X}$, respectively $)$ whenever $g \in \mathcal{X}$. The respective classes are denoted by $\mathcal{M}_{a}(\mathcal{X}), \mathcal{M}_{m}(\mathcal{X})$ and $\mathcal{M}_{l}(\mathcal{X})$. Those classes for some families of Darboux-like functions from $\mathbb{R}$ to $\mathbb{R}$ were studied by several authors. (See, e.g., [Ra] and $[\mathrm{Fa}]$ for the class of all Darboux functions, $[\mathrm{AB}]$ and $[\mathrm{RF}]$ for the class of all Darboux, Baire one functions, $[\mathrm{KB}]$ for the class of all perfect road functions and for the class of all peripherally continuous functions and [JJN] for the class of almost 
continuous functions, for the class of connectivity functions and for the class of functionally connected functions. See also the survey [GN] for definitions and relations between those properties.) The first systematic study of the operators $\mathcal{M}_{a}\left({ }_{-}\right), \mathcal{M}_{m}\left({ }_{-}\right)$and $\mathcal{M}_{l}\left({ }_{-}\right)$was done by Jastrzȩbski, Jȩdrzejewski and Natkaniec in [JJN]. In particular, they proved the following two basic lemmas.

Lemma 1.1. ([JJN, Lemma 2.1]) Let $\Phi$ be a property of functions and $X$ be a topological space. For $i=1,2$ let $\mathcal{X}_{i}$ be the class of all functions $f: X \rightarrow \mathbb{R}^{i}$ with property $\Phi$. Suppose the classes $\mathcal{X}_{1}$ and $\mathcal{X}_{2}$ fulfill the following conditions.

(1.1) If $g \in \mathcal{X}_{2}$ and $h \in \mathrm{C}\left(\mathbb{R}^{2}, \mathbb{R}\right)$, then $h \circ g \in \mathcal{X}_{1}$.

(1.2) If $f \in \mathcal{X}_{1}$ and $g \in \mathrm{C}(X, \mathbb{R})$, then $(f, g) \in \mathcal{X}_{2}$.

Then $\mathrm{C}(X, \mathbb{R}) \subset \mathcal{M}_{a}\left(\mathcal{X}_{1}\right) \cap \mathcal{M}_{m}\left(\mathcal{X}_{1}\right) \cap \mathcal{M}_{l}\left(\mathcal{X}_{1}\right)$.

Lemma 1.2. ([JJN, Lemma 2.2]) Let $\mathcal{X}$ be a family of real functions defined on intervals that fulfills the following conditions.

(2.0) If $f \in \mathcal{X}$ and $x$ belongs to the domain of $f$, then the sets $C^{+}(f, x)$, $C^{-}(f, x)$ are connected and $f(x) \in C^{+}(f, x) \cap C^{-}(f, x)$.

(2.1) If $f: I \rightarrow \mathbb{R}, f \in \mathcal{X}$ and $J$ is a subinterval of an interval $I$, then $f\lceil J \in \mathcal{X}$.

(2.2) If $h:(a, b) \rightarrow \mathbb{R}, h \in \mathcal{X}, y \in C^{+}(h, a), z \in C^{-}(h, b)$, then the functions $h_{1}:[a, b) \rightarrow \mathbb{R}, h_{2}:(a, b] \rightarrow \mathbb{R}$ and $h_{3}:[a, b] \rightarrow \mathbb{R}$ belong to $\mathcal{X}$, where $h_{1}=h \cup\{(a, y)\}, h_{2}=h \cup\{(b, z)\}, h_{3}=h_{1} \cup h_{2}$.

(2.3) If $I \subset \mathbb{R}$ is an interval, $a \in I$ and $f \uparrow(I \cap(-\infty, a]) \in \mathcal{X}, f \uparrow(I \cap[a,+\infty)) \in$ $\mathcal{X}$, then $f \in \mathcal{X}$.

(2.4) Const $\subset \mathcal{M}_{a}(\mathcal{X})$ and $-1 \in \mathcal{M}_{m}(\mathcal{X})$.

(2.5) If $f: I \rightarrow(0, \infty)$ and $f \in \mathcal{X}$, then $1 / f \in \mathcal{X}$.

Then

(i) $\mathcal{M}_{a}(\mathcal{X}) \cup \mathcal{M}_{l}(\mathcal{X}) \subset \mathrm{C}$;

(ii) $\mathcal{M}_{m}(\mathcal{X}) \subset \mathrm{M}$.

We shall employ those lemmas for description of the maximal additive, multiplicative and lattice-like classes for the family of all extendable functions. In our study we shall use the characterization of extendable functions via families of peripheral intervals. (See $[\mathrm{GR}]$.) 
Definition 1. Let $f: \mathbb{I} \rightarrow \mathbb{I}$ be a function. A family of peripheral intervals (or simply, a PI family) for $f$ consists of a sequence of ordered pairs $\left(I_{n}, J_{n}\right)$ of subintervals of $\mathbb{I}$ such that

(1) $I_{n}$ is open in $\mathbb{I}$ and the length of $I_{n}$ converges to 0;

(2) for each $x \in \mathbb{I}$ and for any $\varepsilon>0$ there exists $\left(I_{n}, J_{n}\right)$ such that $x \in I_{n}$, $f(x) \in J_{n}$ and the length of $I_{n}$ and $J_{n}$ are less than $\varepsilon$;

(3) both endpoints of $I_{n}$ map into $J_{n}$;

(4) if $I_{n}$ and $I_{m}$ have points in common but neither is a subset of the other, then $J_{n}$ and $J_{m}$ have points in common.

Gibson and Roush in [GR, Theorems 1 and 2] proved the following theorem.

Theorem 1.1. If $f: \mathbb{I} \rightarrow \mathbb{I}$ is an extendable function, then there exists a PI family for $f$. On the other hand, if for $f: \mathbb{I} \rightarrow \mathbb{I}$ there exists a PI family, then $f$ is an extendable function. Moreover, then $f$ is the restriction of connectivity function $F: \mathbb{I}^{2} \rightarrow \mathbb{I}$ such that $F$ is continuous on the complement of $\mathbb{I} \times\{0\}$.

It is easy to observe that the analogous characterization is valid for any real function defined on an interval.

\section{Extendable Functions}

Lemma 2.1. If $g \in \mathrm{PC}(X, Y)$ and $h \in \mathrm{C}(Y, Z)$, then $h \circ g \in \mathrm{PC}(X, Z)$.

Proof. Let $x \in X, U$ and $V$ be open neighborhoods of $x$ and $h(g(x))$, respectively. Then there exists an open neighborhood $W \subset Y$ of the point $g(x)$ such that $h(W) \subset V$ and there exists an open neighborhood $U_{0} \subset U$ of the point $x$ such that $g\left(\operatorname{bd} U_{0}\right) \subset W$. Hence $h\left(g\left(\operatorname{bd} U_{0}\right)\right) \subset V$.

Corollary 2.1. If $g \in \mathrm{E}(X, Y)$ and $h \in \mathrm{C}(Y, Z)$, then $h \circ g \in \mathrm{E}(X, Z)$.

Proof. There exists a peripherally continuous function $G: X \times \mathbb{I} \rightarrow Y$ such that $g(x)=G(x, 0)$ for all $x \in X$. Then, by Lemma 2.1, the function $h \circ$ $G: X \times \mathbb{I} \rightarrow Z$ is peripherally continuous, and $h \circ g(x)=h \circ G(x, 0)$ for all $x \in X$. So $h \circ g$ has property $\mathcal{E}$.

Lemma 2.2. Assume that $X$ is a regular topological space. If $f \in \operatorname{PC}(X, Y)$ and $g \in \mathrm{C}(X, Z)$, then $(f, g) \in \mathrm{PC}(X, Y \times Z)$. 
Proof. Fix $x_{0} \in X$. Let $U$ be an open neighborhood of $x_{0}$ and $V$ be an open neighborhood of $\left(f\left(x_{0}\right), g\left(x_{0}\right)\right)$. There exist open neighborhoods $V_{1} \subset Y$ and $V_{2} \subset Z$ of $f\left(x_{0}\right)$ and $g\left(x_{0}\right)$, respectively, such that $V_{1} \times V_{2} \subset V$. Let $U_{1}$ be an open subset of $U$ such that $x_{0} \in U_{1}, g\left(U_{1}\right) \subset V_{2}$ and let $U_{2}$ be an open set such that $\overline{U_{2}} \subset U_{1}, x_{0} \in U_{2}$ and $f\left(\operatorname{bd} U_{2}\right) \subset V_{1}$. Hence

$$
(f, g)\left(\operatorname{bd} U_{2}\right) \subset f\left(\operatorname{bd} U_{2}\right) \times g\left(\operatorname{bd} U_{2}\right) \subset V_{1} \times V_{2} \subset V .
$$

Thus $(f, g)$ is a peripherally continuous function at $x_{0}$.

Corollary 2.2. Assume that $X$ is a regular topological space. If $f \in \mathrm{E}(X, Y)$ and $g \in \mathrm{C}(X, Z)$, then $(f, g) \in \mathrm{E}(X, Y \times Z)$.

Now, Corollaries 2.1, 2.2 and Lemma 1.1 imply the following inclusions.

\section{Corollary 2.3.}

$$
\mathrm{C}(\mathbb{R}, \mathbb{R}) \subset \mathcal{M}_{a}(\mathrm{E}(\mathbb{R}, \mathbb{R})) \cap \mathcal{M}_{m}(\mathrm{E}(\mathbb{R}, \mathbb{R})) \cap \mathcal{M}_{l}(\mathrm{E}(\mathbb{R}, \mathbb{R}))
$$

\section{Corollary 2.4.}

$$
\mathrm{C}(\mathbb{R}, \mathbb{R}) \subset \mathcal{M}_{a}(\operatorname{Ext}(\mathbb{R}, \mathbb{R})) \cap \mathcal{M}_{m}(\operatorname{Ext}(\mathbb{R}, \mathbb{R})) \cap \mathcal{M}_{l}(\operatorname{Ext}(\mathbb{R}, \mathbb{R}))
$$

Now we shall verify that the class of all extendable real functions satisfies all assumptions of Lemma 1.2.

Lemma 2.3. Assume that $g:(c, \infty) \rightarrow \mathbb{R}$ is an extendable function and $y \in$ $C^{+}(g, c) \cap \mathbb{R}$. Then $f=g \cup\{(c, y)\}$ is also an extendable function.

Proof. Let $\mathcal{J}_{0}$ be a PI family for $g$. For every $n \in \mathbb{N}$ choose $c_{n} \in(c, c+1]$ such that

(a) $c_{0}=c+1$

(b) $c_{n}<\min \left(c+\frac{1}{n}, c_{n-1}\right)$;

(c) $\left|g\left(c_{n}\right)-y\right|<\frac{1}{n}$.

Put $C=\left\{c_{n}: n \in \mathbb{N}\right\} \cup\{c\}$. Now we shall construct a PI family $\mathcal{J}$ for $f$. A pair $(I, J)$ belongs to $\mathcal{J}$ iff either

(i) $I=\left[c, c_{n}\right)$ and $J=\left(y-\frac{1}{n}, y+\frac{1}{n}\right)$ for some $n \in \mathbb{N}$; or

(ii) $(I, J) \in \mathcal{J}_{0}, I \cap C=\left\{c_{m}\right\}$ and $g\left(c_{m}\right) \in J$; or

(iii) $(I, J) \in \mathcal{J}_{0}$ and $C \cap I=\emptyset$. 
We shall verify that $\mathcal{J}$ is a PI family for $f$. Arrange all elements of $\mathcal{J}$ in a sequence $\left(I_{n}, J_{n}\right)_{n \in N}$. Then all $I_{n}$ are open in $[c, \infty)$ and the lengths of $I_{n}$ converge to 0; so condition (1) from Definition 1 is satisfied.

For $x=c$ condition (2) is clear by (i). For $x \neq c,(2)$ follows easily from the fact that $\mathcal{J}_{0}$ is a PI family for $g$.

Condition (3) is also obvious. Thus we have to verify only condition (4). Fix $n, m$ such that $I_{n} \cap I_{m} \neq \emptyset$ and neither is a subset of the other. Note that either $c \notin I_{n}$ or $c \notin I_{m}$. If $c \notin I_{n} \cup I_{m}$, then $\left(I_{n}, J_{n}\right),\left(I_{m}, J_{m}\right) \in \mathcal{J}_{0}$ and therefore $J_{n} \cap J_{m} \neq \emptyset$. So, suppose that $c \in I_{n}$ and $c \notin I_{m}$. Then $I_{n}=\left[c, c_{k_{n}}\right)$ for some $k_{n} \in N$ and $c_{k_{n}} \in I_{m}$. By (ii), $g\left(c_{k_{n}}\right) \in J_{m}$ and, by (i) and (c), $g\left(c_{k_{n}}\right) \in J_{n}$. Thus $J_{n} \cap J_{m} \neq \emptyset$.

Lemma 2.4. If $c \in \mathbb{R}, f: \mathbb{R} \rightarrow \mathbb{R}$ and $f \uparrow(-\infty, c], f \uparrow[c, \infty)$ are extendable functions, then $f$ is also an extendable function.

Proof. Let $\mathcal{J}_{0}$ and $\mathcal{J}_{1}$ denote PI families for $f \uparrow(-\infty, c]$ and $f \uparrow[c, \infty)$ respectively. For every $n \in \mathbb{N}$ choose $\left(I_{n}^{-}, J_{n}^{-}\right) \in \mathcal{J}_{0},\left(I_{n}^{+}, J_{n}^{+}\right) \in \mathcal{J}_{1}$ such that $c \in I_{n}^{-} \cap I_{n}^{+}$and the lengths of $I_{n}^{-} \cup I_{n}^{+}$and $J_{n}^{-} \cup J_{n}^{+}$are less than $\frac{1}{n}$. Now we shall define a PI family $\mathcal{J}$ for $f$. A pair $(I, J)$ belongs to $\mathcal{J}$ iff either

(i) $(I, J) \in \mathcal{J}_{0} \cup \mathcal{J}_{1}$ and $c \notin I$; or

(ii) $I=I_{n}^{-} \cup I_{n}^{+}$and $J=J_{n}^{-} \cup J_{n}^{+}$for some $n \in \mathbb{N}$.

It is easy to verify that $\mathcal{J}$ is a PI family for $f$.

From Lemmas 1.2, 2.3 and 2.4 we obtain the following inclusions.

Corollary 2.5.

$$
\begin{aligned}
\mathcal{M}_{a}(\operatorname{Ext}(\mathbb{R}, \mathbb{R})) \cup \mathcal{M}_{l}(\operatorname{Ext}(\mathbb{R}, \mathbb{R})) & \subset \mathrm{C}(\mathbb{R}, \mathbb{R}) \\
\mathcal{M}_{m}(\operatorname{Ext}(\mathbb{R}, \mathbb{R})) & \subset \mathrm{M}
\end{aligned}
$$

Thus, Corollaries 2.4 and 2.5 yield the following equalities:

\section{Theorem 2.1.}

$$
\mathcal{M}_{a}(\operatorname{Ext}(\mathbb{R}, \mathbb{R}))=\mathrm{C}(\mathbb{R}, \mathbb{R})=\mathcal{M}_{l}(\operatorname{Ext}(\mathbb{R}, \mathbb{R}))
$$

Lemma 2.5. Assume that $C$ is a nowhere dense closed subset of $\mathbb{R}$ and $g: \mathbb{R} \rightarrow$ $\mathbb{R}$ satisfies

(a) $g(x)=0$ for $x \in C$;

(b) if $J \subset \mathbb{R}$ is a component of $\mathbb{R} \backslash C$, then $g \nmid \bar{J}$ is an extendable function. 
Then $g$ is an extendable function.

Proof. Let $\left\{\left(a_{n}, b_{n}\right)\right\}_{n}$ be the sequence of all components of $\mathbb{R} \backslash C$. For each $n$ let $\mathcal{K}_{n}$ be a PI family for $g \widehat{\left(a_{n}, b_{n}\right)}$. For every positive integer $n$ choose a finite family $\mathcal{I}_{n}$ of open intervals such that

- $C \subset \cup \mathcal{I}_{n}$;

- the length of each $I \in \mathcal{I}_{n}$ is less than $1 / n$;

- the end-points of every $I \in \mathcal{I}_{n}$ belong to $I \backslash C$;

- if $I \in \mathcal{I}_{n}, \inf (I) \in\left(a_{i}, b_{i}\right), \sup (I) \in\left(a_{j}, b_{j}\right), I^{-}=I \cap\left(a_{i}, b_{i}\right]$ and $I^{+}=I \cap\left[a_{j}, b_{j}\right)$, then there are intervals $J^{-}, J^{+}$such that $\left(I^{-}, J^{-}\right) \in \mathcal{K}_{i}$, $\left(I^{+}, J^{+}\right) \in \mathcal{K}_{j}$ and the length of $J^{-} \cup J^{+}$is less than $1 / n$.

We shall define a PI family $\mathcal{J}$ for $g$. A pair $(I, J)$ belongs to $\mathcal{J}$ iff either

(i) there exists $n$ such that $(I, J) \in \mathcal{K}_{n}$ and $I \cap C=\emptyset$; or

(ii) $I \in \mathcal{I}_{n}$ for some $n$ and $J=J^{-} \cup J^{+}$, where $J^{-}, J^{+}$are described above.

Now we shall verify that $\mathcal{J}$ is a PI family for $g$. We can arrange all elements of $\mathcal{J}$ in a sequence $\left\{\left(I_{n}, J_{n}\right)\right\}_{n}$ such that the lengths of $I_{n}$ converge to 0 ; so condition (1) from Definition 1 is satisfied.

To prove condition (2), fix $x \in \mathbb{R}$ and $\varepsilon>0$. If $x \notin C$, then $x \in\left(a_{m}, b_{m}\right)$ for some $m$. There exists $(I, J) \in \mathcal{K}_{m}$ that fulfills (2) and, by (i), $(I, J) \in \mathcal{J}$. If $x \in C$, then there exist $n \in \mathbb{N}$ and $(I, J) \in \mathcal{J}$ such that $I \in \mathcal{I}_{n}, 1 / n<\varepsilon$ and $x \in I$. Then $g(x)=0 \in J$ and the lengths of $I$ and $J$ are less than $1 / n$; so the pair $(I, J)$ satisfies (2).

Statement (3) is obvious by the definition of $\mathcal{J}$.

To verify (4), fix $\left(I_{n}, J_{n}\right)$ and $\left(I_{m}, J_{m}\right)$ such that $I_{n} \cap I_{m} \neq \emptyset$ and neither is a subset of the other. Note that $0 \in J_{k}$ whenever $I_{k} \cap C \neq \emptyset$. Thus, if $I_{n} \cap C \neq \emptyset \neq I_{m} \cap C$, then $0 \in J_{n} \cap J_{m}$. If $\left(I_{n} \cup I_{m}\right) \cap C=\emptyset$, then $I_{n} \cup I_{m} \subset\left(a_{i}, b_{i}\right)$ for some $i \in \mathbb{N}$ and therefore $\left(I_{n}, J_{n}\right),\left(I_{m}, J_{m}\right) \in \mathcal{K}_{i}$. Thus $J_{n} \cap J_{m} \neq \emptyset$. So, assume that $I_{n} \cap C \neq \emptyset$ and $I_{m} \cap C=\emptyset$. Then $I_{m} \subset\left(a_{i}, b_{i}\right)$ for some $i \in \mathbb{N}$ and either $a_{i} \in I_{n}$ or $b_{i} \in I_{n}$. Suppose that $b_{i} \in I_{n}$. By the definition of $\mathcal{J}$ there exist $\left(I_{0}, J_{0}\right) \in \mathcal{K}_{i}$ such that $I_{0}=I_{n} \cap\left(a_{i}, b_{i}\right]$ and $J_{0} \subset J_{n}$. Because $\left(I_{m}, J_{m}\right),\left(I_{0}, J_{0}\right) \in \mathcal{K}_{i}$ and $I_{m} \cap I_{0} \neq \emptyset$, we get $J_{m} \cap J_{0} \neq \emptyset$. Consequently, $J_{m} \cap J_{n} \neq \emptyset$; so $\mathcal{J}$ satisfies condition (4).

\section{Theorem 2.2.}

$$
\mathcal{M}_{m}(\operatorname{Ext}(\mathbb{R}, \mathbb{R}))=\mathrm{M}
$$


Proof. By Corollary 2.5, it is enough to prove that $\mathrm{M} \subset \mathcal{M}_{m}(\operatorname{Ext}(\mathbb{R}, \mathbb{R}))$. Assume that $f \in \operatorname{Ext}(\mathbb{R}, \mathbb{R})$ and $g \in \mathrm{M}$. Then $D=\operatorname{bd}\left(f^{-1}(0)\right)$ is a closed and nowhere dense set. Let $J$ be a component of the complement of $D$. Then $g$ is continuous on $\bar{J}$ and, by Corollary 2.4, $f g$ is extendable on $\bar{J}$. Moreover, $f g(x)=0$ for $x \in D$, and according to Lemma 2.5, $f g$ is an extendable function.

\section{Applications}

The next lemma shows that in the definition of extendability (for real functions) we can replace the compact interval II by whole real line.

Lemma 3.1. For a function $f: \mathbb{R} \rightarrow \mathbb{R}$ the following conditions are equivalent.

(i) $f \in \operatorname{Ext}(\mathbb{R}, \mathbb{R})$.

(ii) there is $F \in \mathrm{PC}\left(\mathbb{R}^{2}, \mathbb{R}\right)$ such that $f(x)=F(x, 0)$ for each $x \in \mathbb{R}$.

Proof. Let $f \in \operatorname{Ext}(\mathbb{R}, \mathbb{R})$. Then there exists $\left.F_{0} \in \mathrm{PC}(\mathbb{R} \times \mathbb{I}, \mathbb{R})\right)$ such that $f(x)=F_{0}(x, 0)$ for $x \in \mathbb{R}$. According to [GR, Theorem 2], we can assume that $F_{0}$ is continuous on $\mathbb{R} \times(0,1]$. Thus $F_{+}: \mathbb{R} \times[0, \infty) \rightarrow \mathbb{R}$ defined by

$$
F_{+}(x, y)= \begin{cases}F_{0}(x, y) & \text { for }(x, y) \in \mathbb{R} \times \mathbb{I} \\ F_{0}(x, 1) & \text { for }(x, y) \in \mathbb{R} \times[1, \infty)\end{cases}
$$

is a peripherally continuous function and consequently $F: \mathbb{R}^{2} \rightarrow \mathbb{R}$ defined by

$$
F(x, y)= \begin{cases}F_{+}(x, y) & \text { for }(x, y) \in \mathbb{R} \times[0, \infty) \\ F_{+}(x,-y) & \text { for }(x, y) \in \mathbb{R} \times(-\infty, 0]\end{cases}
$$

is also a peripherally continuous function with $f(x)=F(x, 0)$ for every $x \in \mathbb{R}$.

Now, if $F \in \operatorname{PC}\left(\mathbb{R}^{2}, \mathbb{R}\right)$ and $f(x)=F(x, 0)$ for each $x \in \mathbb{R}$, then $F \uparrow(\mathbb{R} \times \mathbb{I})$ is also a peripherally continuous extension of $f$. Thus $f \in \operatorname{Ext}(\mathbb{R}, \mathbb{R})$.

\section{Theorem 3.1.}

$$
\mathcal{M}_{a}\left(\operatorname{PC}\left(\left(\mathbb{R}^{2}, \mathbb{R}\right)\right)=\mathrm{C}\left(\mathbb{R}^{2}, \mathbb{R}\right)=\mathcal{M}_{l}\left(\mathrm{PC}\left(\mathbb{R}^{2}, \mathbb{R}\right)\right)\right.
$$

Proof. By Lemmas 2.1 and $2.2, \mathrm{C}\left(\mathbb{R}^{2}, \mathbb{R}\right) \subset \mathcal{M}_{a}\left(\mathrm{PC}\left(\mathbb{R}^{2}, \mathbb{R}\right)\right)$. On the other hand, $\mathcal{M}_{a}\left(\mathrm{PC}\left(\mathbb{R}^{2}, \mathbb{R}\right)\right) \subset \mathrm{PC}\left(\mathbb{R}^{2}, \mathbb{R}\right)$ because $f \equiv 0$ belongs to $\mathrm{PC}\left(\mathbb{R}^{2}, \mathbb{R}\right)$. Suppose that $g \in \operatorname{PC}\left(\mathbb{R}^{2}, \mathbb{R}\right)$ is discontinuous at $x_{0} \in \mathbb{R}^{2}$. Let $h: \mathbb{R} \rightarrow \mathbb{R}^{2}$ be a homeomorphic injection of $\mathbb{R}$ into $\mathbb{R}^{2}$ such that 
- $h(0)=x_{0}$ and $g \circ h$ is discontinuous at 0 ;

- there is a homeomorphism $h_{1}: \mathbb{R}^{2} \rightarrow \mathbb{R}^{2}$ such that $h_{1}(x, 0)=h(x)$ for $x \in \mathbb{R}$.

By Corollary 2.5, there is $f_{0} \in \operatorname{Ext}(\mathbb{R}, \mathbb{R})$ such that $f_{0}+g \circ h \notin \operatorname{Ext}(\mathbb{R}, \mathbb{R})$. Let $f_{1}: \mathbb{R}^{2} \rightarrow \mathbb{R}$ be a peripherally continuous extension of $f_{0}$, i.e., $f_{1}(x, 0)=f_{0}(x)$ for $x \in \mathbb{R}$. Then $f=f_{1} \circ h_{1}^{-1} \in \operatorname{PC}\left(\mathbb{R}^{2}, \mathbb{R}\right)$. Suppose that $f+g \in \operatorname{PC}\left(\mathbb{R}^{2}, \mathbb{R}\right)$. Then

$$
f_{1}+g \circ h_{1}=(f+g) \circ h_{1} \in \mathrm{PC}\left(\mathbb{R}^{2}, \mathbb{R}\right) .
$$

On the other hand, for each $x \in \mathbb{R}$ we have

$$
\left(f_{1}+g \circ h_{1}\right)(x, 0)=f_{1}(x, 0)+g\left(h_{1}(x, 0)\right)=f_{0}(x)+g \circ h(x) .
$$

Thus $f_{0}+g \circ h \in \operatorname{Ext}(\mathbb{R}, \mathbb{R})$, contrary to the choice of $f_{0}$. Consequently,

$$
\mathcal{M}_{a}\left(\mathrm{PC}\left(\mathbb{R}^{2}, \mathbb{R}\right)\right) \subset \mathrm{C}\left(\mathbb{R}^{2}, \mathbb{R}\right) .
$$

In a similar way we can prove that

$$
\mathcal{M}_{l}\left(\mathrm{PC}\left(\mathbb{R}^{2}, \mathbb{R}\right)\right)=\mathrm{C}\left(\mathbb{R}^{2}, \mathbb{R}\right) .
$$

To see it, note that $\mathcal{M}_{l}\left(\mathrm{PC}\left(\mathbb{R}^{2}, \mathbb{R}\right)\right) \subset \mathrm{PC}\left(\mathbb{R}^{2}, \mathbb{R}\right)$. Indeed, suppose that $f: \mathbb{R}^{2} \rightarrow$ $\mathbb{R}$ is not peripherally continuous at $x_{0} \in \mathbb{R}^{2}$. Then there is an open neighborhood $W$ of $x_{0}$ and $\varepsilon>0$ such that $f(\operatorname{bd}(U)) \subset\left(f\left(x_{0}\right)-\varepsilon, f\left(x_{0}\right)+\varepsilon\right)$ for no open neighborhood $U$ of $x_{0}$ with $U \subset W$. Then the function $\max \left(f, f\left(x_{0}\right)-\varepsilon\right)$ is not peripherally continuous at $x_{0}$ and the constant function $f\left(x_{0}\right)-\varepsilon$ is peripherally continuous. Thus $f \notin \mathcal{M}_{l}\left(\mathrm{PC}\left(\mathbb{R}^{2}, \mathbb{R}\right)\right)$.

Finally, note that Lemmas 2.1 and 2.2 yield $\mathrm{C}\left(\mathbb{R}^{2}, \mathbb{R}\right) \subset \mathcal{M}_{m}\left(\mathrm{PC}\left(\mathbb{R}^{2}, \mathbb{R}\right)\right)$. On the other hand, it is easy to verify that $f: \mathbb{R}^{2} \rightarrow \mathbb{R}$ defined by

$$
f(x)= \begin{cases}\sin \left(\|x\|^{-1}\right) & \text { if } x \neq 0 \\ 0 & \text { if } x=0\end{cases}
$$

is a discontinuous function that belongs to $\mathcal{M}_{m}\left(\mathrm{PC}\left(\mathbb{R}^{2}, \mathbb{R}\right)\right)$. Thus we finish with the following problem:

Problem 1. Characterize the class $\mathcal{M}_{m}\left(\mathrm{PC}\left(\mathbb{R}^{2}, \mathbb{R}\right)\right)$. 


\section{References}

[KB] K. Banaszewski, Algebraic properties of $\mathcal{E}$-continuous functions, Real Anal. Exchange 18 (1992-93), 153-168.

[BHL] J. Brown, P. Humke and M. Laczkovich, Measurable Darboux functions, Proc. Amer. Math. Soc. 102 (1988), 603-610.

[AB] A. M. Bruckner, Differentiation of Real Functions, Lecture Notes in Mathematics, Vol. 659 Springer-Verlag, Berlin, Heidelberg, New York, 1978.

[Fa] J. Farková, About the maximum and the minimum of Darboux functions, Mat. Cas. Slov. Akad. Vied 21 (1971), 110-116.

[RF] R. J. Fleissner, A note on Baire 1 Darboux functions, Real Anal. Exchange 3 (1977-78), 104-106.

[GN] R. G. Gibson and T. Natkaniec, Darboux like functions, Real Anal. Exchange 22 (1996-97), 492-534.

[GR] R. G. Gibson and F. Roush, A characterization of extendable connectivity functions, Real Anal. Exchange 13 (1987-88), 214-222.

[H] M. R. Hagan, Equivalence of connectivity maps and peripherally continuous transformations, Proc. Amer. Math. Soc. 17 (1966), 175-177.

$[\mathrm{OH}]$ O. H. Hamilton, Fixed points for certain noncontinuous transformations, Proc. Amer. Math. Soc. 8 (1957), 750-756.

[JJN] J. Jastrzębski, J. Jędrzejewski and T. Natkaniec, On some subclasses of Darboux functions, Fund. Math. 138 (1991), 165-173.

[Ra] T. Radaković, Über Darbouxsche und stetige Funktionen, Monat. Math. Phys. 38 (1931), 111-122.

[JS] J. Stallings, Fixed point theorems for connectivity maps, Fund. Math. 47 (1959), 249-263. 